\title{
Railway Network Blocking Problem: An Optimization Modeling Formulation about Flow Routing Problem
}

\author{
Hongpeng $\mathrm{Ma}^{1, \mathrm{a}}$, Yixiang Yue $\mathrm{e}^{2,}$ and Congli $\mathrm{Hao}^{3, \mathrm{c}}$ \\ ${ }^{1}$ School of Traffic and Transportation, Beijing Jiaotong University, Beijing 100044, China \\ ${ }^{2}$ School of Traffic and Transportation, Beijing Jiaotong University, Beijing 100044, China \\ ${ }^{3}$ School of Traffic and Transportation, Beijing Jiaotong University, Beijing 100044, China

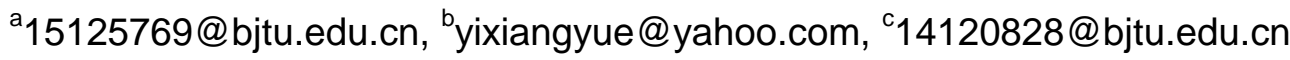

\begin{abstract}
Keywords: Railroad; Road Network; Blocking Problem; Optimization
Abstract: In this paper, we mainly study on modeling formulation for railway network blocking problem. We propose model formulation for RBP. The objective function of RBP model is to minimize the costs of flow traveling and delay for the train in marshalling station, by deciding which block is built and specifying the assignment of commodities to these blocks, while observing limits on the reclassification capacity at each terminal. The model is solved using GAMS. The model is tested on a real-world railway network located in North of China, the computation results show that the model have the potential to apply and can yield the dramatic railroad's operating costs saving.
\end{abstract}

\section{Introduction}

The Railway Blocking Problem (RBP) determines how to aggregate a large number of shipments into blocks of shipments as they travel from origins to destinations [1]. In other words, RBP determines which blocks should be built at each yard and what shipments should be placed in the block.

Mathematically, RBP is a multi-commodity-flow, network-design, and routing problem. To solve RBP, we need to design the underlying blocking network and to route different commodities on the blocking network to minimize the transportation costs [2]. In RBP, each train will be assigned to a direct block, whose OD is the same as that of the shipment, to avoid unnecessary marshalling and delays. So there are some directed arcs between two terminals that are not necessarily connected by a physical link. However, blocking capacity at each yard, determined by available yard resources (hump yard equipment and shunting yard equipment), limits the maximum number of blocks and maximum car volume that each yard can handle, preventing railroads from assigning direct blocks for all trains. So aiming at delivering the flow with the fewest possible classifications, railroads develop RBP determining which blocks should be built at each yard and what shipments should be placed in each block $[3,4,5,6]$.

RBP is one of the most important decision in freight railroads. A good solution of RBP can save railway operation costs of delivering all commodities. And these costs are usually broken down into car-handling costs associated with handling (or blocking) a car and car-miles costs associated with the movement of a car.

There are some study about blocking problem of single railway line, $\mathrm{Xu}$ [7] proposed a 0-1 programming with the target of minimum balanced using of adaptation capacity and hour of freight train in marshalling station. And Yang [8] proposed 0-1 linear programming model and 0-1 quadratic program model.

Compared with single railway line, the reality railroad network is much complicated. For example, number of transport plan about blocking problem in single line railroad is 1048576, but in railway network, the number may be million, even billion. So for complicated railroad network blocking problem, there are very few study in the field. Li [9] proposed Chance Constrained Programming with considering flow, assembly time and volatility of vehicle adaptation extra time consuming. Newton [10] and Newton et al. [11] modeled the blocking problem as a network-design model and formulated it as a MIP. Bodin [12] established Nonlinear Mixed Integer Programming model with the target of 
minimum total cost of adaptation and transport, and proposed heuristic decomposition method. Yaghini [13] and Yue [14] proposed Ant Colony Optimization Algorithm to solve the railroad blocking problem.

Their approaches focus on determining a near-optimal solution. However, many models only solve blocking problem of single railway network, such as 0-1 linear programming model. And the approaches for RBP can't guarantee to get optimal solution in any case without considering factors, such as, empty car, flow pathway and service level. So it is very necessary to develop optimization modeling formulation of RBP.

\section{Model and Solution Method}

Notation. $i, j, n, k, q$ is macroscopic node index. And $i, j, n$ correspond to physical marshalling station in a railway network. $k$ is origin station of commodity and $q$ is destination station of commodity in a railway network. $c_{i, j}$ is flow transport cost between each pair $\mathrm{OD}$ (from station $i$ to station $j$ ), is proportional to mileage between the pair OD. $c_{i, j}$ can be calculated by any empirical formula according to statistical curve fitting. Conversion coefficient that changing car-hour delay to cost is $\mathrm{p}$, and the value of $\mathrm{p}$ is 80 in this study. $m_{i, j}$ is accumulation delay when the directed block to station $j$ is built in station $i$. The empirical formulation is shown as equation (1).

$$
m_{i, j}=\alpha_{i} \times s_{i, j}
$$

Where $\alpha_{i}$ is accumulation parameter of marshalling station $i$, it is a constant derived from statistical analysis by many years record data. $s_{i, j}$ is number of cars for one train from station $i$ to station $j . \boldsymbol{t}_{i}$ is the save time of car passing station $i$ without reclassification. $u_{i, j}$ is re-classification capacity from station $i$ to direction $j . d_{k, q}$ is demand from station $k$ to station $q$. $\mathrm{M}$ is a large number. $b_{i, j}$ is directed-block index as equation (2).

$$
b_{i, j}= \begin{cases}1 & \text { there is directed block from station } i \text { to station } j \quad \forall i, j \\ 0 & \text { otherwise }\end{cases}
$$

There are two decision variables: $x_{i, j, k, q}$ and $y_{i, j} \cdot x_{i, j, k, q}$ is volume from station $k$ to station $q$ shipped using train from station $i$ to station $j . y_{i, j}$ is $0-1$ binary variables as equation (3).

$$
y_{i, j}=\left\{\begin{array}{ll}
1 & \text { there is train from station } i \text { to station } j \\
0 & \text { otherwise }
\end{array} \forall i, j\right.
$$

Formulation of RBP Model. The objective function of RBP model is to minimize the costs of flow traveling and delay for the train in marshalling stations, by deciding which block is built and specifying the assignment of commodities to these blocks, while observing limits on reclassification capacity at each terminal.

The formulation of RBP is as follow.

$$
z=\min \sum_{i, j} \sum_{k, q} x_{i, j, k, q} \times c_{i, j}+p \times\left[\sum_{i, j} m_{i, j} \times y_{i, j} \times b_{i, j}+\sum_{j} t_{j} \times\left(\sum_{i, k, q} x_{i, j, k, q}-\sum_{k} d_{k, j}\right)\right]
$$

Subject to

$$
\begin{array}{ll}
\sum_{i, q} x_{i, j, k, q}-d_{k, j} \leq u_{j, k} & \forall j, k \\
\sum_{j}^{j} x_{i, j, k, q}=d_{k, q} & \forall i, k, q, i=k \\
\sum_{i} x_{i, j, k, q}=d_{k, q} & \forall j, k, q, j=q \\
\sum_{j} x_{i, j, k, q}-\sum_{n} x_{n, i, k, q}=0 & \forall i, k, q, i \neq q, i \neq k \\
x_{i, j, k, q} \leq M y_{i, j} & \forall i, j, k, q
\end{array}
$$


The objective function of RBP model is to minimize the total cost consists of flow transport cost in railroad and delay in marshalling station. Constraint (5) is the hard constraint of reclassification capacity of the number of blocking cars satisfied reclassification capacity in every marshalling station. Constraint (6)、(7) and (8) is flow balance constraint. Constraint (9) ensures that if there is no train from station $i$ to station $j$, volume from station $k$ to station $q$ shipped by train from station $i$ to station $j$ must be zero, which means if $y_{i, j}=0$, there is that $x_{i, j, k, q}=0$.

\section{Real World Case Study}

Railway Network. Based on eight marshalling stations, the railroad network of North China is constructed to calculate as in Fig. 1. All intermediate stations isn't shown in Fig. 1.

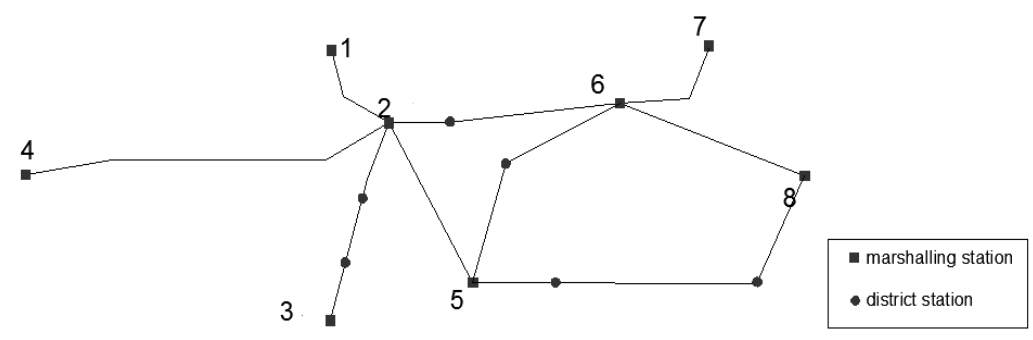

Fig. 1.Case of railroad network

The real world data collected is shown in Table 1, Table 2, Table 3 and Table 4. Station 2 is the center of railway network with five convergence directions, so accumulation delay of station 2 is detailed in Table 2.

\begin{tabular}{ccccccccc}
\hline arrival leave & 1 & 2 & 3 & 4 & 5 & 6 & 7 & 8 \\
\hline 1 & 0 & 84 & 389 & 832 & 324 & 292 & 693 & 609 \\
2 & 84 & 0 & 355 & 798 & 290 & 258 & 659 & 575 \\
3 & 389 & 355 & 0 & 1103 & 465 & 563 & 964 & 874 \\
4 & 832 & 798 & 1103 & 0 & 1038 & 1006 & 1407 & 1323 \\
5 & 324 & 290 & 465 & 1038 & 0 & 430 & 831 & 536 \\
6 & 292 & 258 & 563 & 1006 & 430 & 0 & 451 & 367 \\
7 & 704 & 659 & 964 & 1537 & 831 & 451 & 0 & 402 \\
8 & 609 & 575 & 874 & 1323 & 536 & 367 & 402 & 0 \\
\hline
\end{tabular}

Table 1. Flow transport cost between each pair OD [\$/car]

\begin{tabular}{cccccccc}
\hline departure & 1 & 3 & 4 & 5 & 6 & 7 & 8 \\
\hline Number of cars of one train of station B[car] & 49.3 & 49.3 & 52.6 & 42 & 41.8 & 50.5 & 52.3 \\
Accumulation Parameter of station B[h/car] & 9.1 & 10.1 & 8.2 & 9 & 9.3 & 10.6 & 4.1 \\
Accumulation delay of station B[h] & 449 & 498 & 431 & 378 & 389 & 535 & 215 \\
\hline
\end{tabular}

Table 2. Accumulation delay of station 2

\begin{tabular}{ccccccccccc}
\hline \multicolumn{2}{c}{ station } & & 1 & 3 & 4 & 5 & 6 & 7 & 8 \\
\hline \multicolumn{2}{c}{ Accumulation delay of other stations $[\mathrm{h}]$} & 550 & 550 & 636 & 600 & 500 & 530 & 530 \\
\hline \multicolumn{2}{c}{ Table 3. Accumulation delay of other marshalling stations } \\
\hline \multicolumn{2}{c}{ station } & 1 & 2 & 3 & 4 & 5 & 6 & 7 & 8 \\
\hline $\mathrm{t}_{\mathrm{i}}[\mathrm{h} / \mathrm{car}]$ & 3 & 2.9 & 4 & 4.7 & 3 & 3 & 4 & 4 \\
\multirow{2}{*}{$\begin{array}{c}\text { reclassification } \\
\text { capacity[car] }\end{array}$} & Station 2 & 321 & - & 651 & 1110 & 39 & 1125 & 1125 & 1125 \\
& Station 6 & 1125 & 1125 & 1125 & 1125 & 88 & - & 700 & 1100 \\
& Station 5 & 500 & 500 & 500 & 500 & - & 500 & 500 & 30 \\
\hline
\end{tabular}

Table 4. The value of parameter for marshalling station

Model Testing and Result Analysis. We use General Algebraic Modeling System (GAMS) [15] to solve MIP model of RBP. And calculation time of RBP solved by GAMS are 30 seconds. 
To verify feasibility of model of RBP, we solve respectively the RBP with real flow data in 2013 and 2014. And the traffic demand between of each pair OD in 2013 and 2014 is shown in Table 5 and Table 6.

\begin{tabular}{rccccccccc}
\hline Arrival & leave & 1 & 2 & 3 & 4 & 5 & 6 & 7 & 8 \\
\hline 1 & 0 & 190 & 70 & 125 & 110 & 10 & 20 & 25 \\
2 & 150 & 0 & 245 & 20 & 24 & 300 & 153 & 385 \\
3 & 272 & 442 & 0 & 160 & 17 & 140 & 130 & 29 \\
4 & 85 & 405 & 150 & 0 & 9 & 135 & 50 & 185 \\
5 & 59 & 140 & 4 & 3 & 0 & 4 & 3 & 0 \\
6 & 15 & 35 & 230 & 120 & 40 & 0 & 50 & 32 \\
7 & 11 & 221 & 282 & 138 & 26 & 31 & 0 & 0 \\
8 & 40 & 30 & 50 & 490 & 4 & 57 & 0 & 0 \\
\hline Arrival & leave & 1 & 2 & 3 & 4 & 5 & 6 & 7 & 8 \\
\hline 1 & 0 & 190 & 67 & 125 & 106 & 13 & 9 & 34 \\
2 & 161 & 0 & 231 & 22 & 24 & 315 & 153 & 396 \\
3 & 72 & 442 & 0 & 161 & 17 & 131 & 160 & 29 \\
4 & 89 & 401 & 293 & 0 & 9 & 132 & 49 & 185 \\
5 & 59 & 135 & 4 & 3 & 0 & 4 & 3 & 0 \\
6 & 15 & 36 & 231 & 360 & 40 & 0 & 50 & 32 \\
7 & 11 & 21 & 282 & 134 & 26 & 30 & 0 & 0 \\
8 & 38 & 30 & 50 & 505 & 4 & 59 & 0 & 0 \\
\hline
\end{tabular}

Table 6. Traffic demand between each pair OD in 2014 [car]

We use GAMS to solve model of RBP. And the solution is shown in Table 7, Table 8, Table 9 and Table 10.

\begin{tabular}{cccccccccccccccccccc}
\hline$i$ & $j$ & $k$ & $q$ & $x_{i, j, k, q}$ & $i$ & $j$ & $k$ & $q$ & $x_{i, j, k, q}$ & $i$ & $j$ & $k$ & $q$ & $x_{i, j, k, q}$ & $i$ & $j$ & $k$ & $q$ & $x_{i, j, k, q}$ \\
\hline 1 & 2 & 1 & 2 & 150 & 2 & 4 & 2 & 4 & 405 & 3 & 2 & 3 & 4 & 150 & 6 & 2 & 6 & 1 & 13 \\
1 & 2 & 1 & 4 & 85 & 2 & 4 & 3 & 4 & 150 & 3 & 2 & 3 & 5 & 4 & 6 & 2 & 6 & 2 & 300 \\
1 & 2 & 1 & 5 & 59 & 2 & 4 & 5 & 4 & 9 & 3 & 2 & 3 & 8 & 50 & 6 & 2 & 6 & 3 & 140 \\
1 & 2 & 1 & 6 & 15 & 2 & 4 & 6 & 4 & 135 & 3 & 6 & 3 & 6 & 230 & 6 & 2 & 6 & 4 & 135 \\
1 & 2 & 1 & 7 & 11 & 2 & 4 & 7 & 4 & 50 & 3 & 7 & 3 & 7 & 282 & 6 & 5 & 6 & 5 & 4 \\
1 & 2 & 1 & 8 & 40 & 2 & 5 & 1 & 5 & 59 & 4 & 2 & 4 & 1 & 125 & 6 & 5 & 7 & 5 & 3 \\
1 & 3 & 1 & 3 & 272 & 2 & 5 & 2 & 5 & 140 & 4 & 2 & 4 & 2 & 20 & 6 & 7 & 5 & 7 & 26 \\
2 & 1 & 2 & 1 & 190 & 2 & 5 & 3 & 5 & 4 & 4 & 2 & 4 & 3 & 160 & 6 & 7 & 6 & 7 & 31 \\
2 & 1 & 3 & 1 & 70 & 2 & 5 & 4 & 5 & 3 & 4 & 2 & 4 & 5 & 3 & 6 & 8 & 6 & 8 & 57 \\
2 & 1 & 4 & 1 & 125 & 2 & 6 & 1 & 6 & 15 & 4 & 6 & 4 & 6 & 120 & 7 & 2 & 7 & 1 & 20 \\
2 & 1 & 5 & 1 & 14 & 2 & 6 & 2 & 6 & 35 & 4 & 6 & 4 & 7 & 134 & 7 & 2 & 7 & 2 & 153 \\
2 & 1 & 6 & 1 & 13 & 2 & 6 & 4 & 6 & 120 & 4 & 8 & 4 & 8 & 490 & 7 & 2 & 7 & 3 & 130 \\
2 & 1 & 7 & 1 & 20 & 2 & 7 & 1 & 7 & 11 & 5 & 1 & 5 & 1 & 96 & 7 & 2 & 7 & 4 & 50 \\
2 & 1 & 8 & 1 & 25 & 2 & 7 & 2 & 7 & 221 & 5 & 2 & 5 & 1 & 14 & 7 & 6 & 7 & 5 & 3 \\
\hline$i$ & $j$ & $k$ & 9 & $x_{i, j, k, q}$ & $i$ & $j$ & $k$ & $q$ & $x_{i, j, k, q}$ & $i$ & $j$ & $k$ & $q$ & $x_{i, j, k, q}$ & $i$ & $j$ & $k$ & $q$ & $x_{i, j, k, q}$ \\
\hline 2 & 3 & 2 & 3 & 442 & 2 & 7 & 4 & 7 & 134 & 5 & 2 & 5 & 2 & 24 & 7 & 6 & 7 & 6 & 50 \\
2 & 3 & 4 & 3 & 160 & 2 & 8 & 1 & 8 & 40 & 5 & 2 & 5 & 3 & 17 & 8 & 2 & 8 & 1 & 25 \\
2 & 3 & 5 & 3 & 17 & 2 & 8 & 2 & 8 & 30 & 5 & 2 & 5 & 4 & 9 & 8 & 2 & 8 & 2 & 385 \\
2 & 3 & 6 & 3 & 140 & 2 & 8 & 3 & 8 & 50 & 5 & 6 & 5 & 6 & 40 & 8 & 2 & 8 & 3 & 29 \\
2 & 3 & 7 & 3 & 130 & 3 & 2 & 3 & 1 & 70 & 5 & 6 & 5 & 7 & 26 & 8 & 4 & 8 & 4 & 185 \\
2 & 3 & 8 & 3 & 29 & 3 & 2 & 3 & 2 & 245 & 5 & 8 & 5 & 8 & 4 & 8 & 6 & 8 & 6 & 32 \\
2 & 4 & 1 & 4 & 85 & - & - & - & - & - & - & - & - & - & - & - & - & - & - & - \\
\hline
\end{tabular}

Table 7. The Solution of RBP for variable $x_{i, j, k, q}$ with real flow OD in 2013 


\begin{tabular}{cccccccccc}
\hline$i$ & $j$ & 1 & 2 & 3 & 4 & 5 & 6 & 7 & 8 \\
\hline 1 & 0 & 1 & 1 & 0 & 0 & 0 & 0 & 0 \\
2 & 1 & 0 & 1 & 1 & 1 & 1 & 1 & 1 \\
3 & 0 & 1 & 0 & 0 & 0 & 1 & 1 & 0 \\
4 & 0 & 1 & 0 & 0 & 0 & 0 & 0 & 1 \\
5 & 1 & 1 & 0 & 0 & 0 & 1 & 0 & 1 \\
6 & 0 & 1 & 0 & 0 & 1 & 0 & 1 & 1 \\
7 & 0 & 1 & 0 & 0 & 0 & 1 & 0 & 0 \\
8 & 0 & 1 & 0 & 1 & 0 & 1 & 0 & 0 \\
\hline
\end{tabular}

Table 8. The solution of RBP for variable $y_{i, j}$ with real flow OD in 2013

The objective function value is:

\begin{tabular}{|c|c|c|c|c|c|c|c|c|c|c|c|c|c|c|c|c|c|c|c|}
\hline$i$ & $j$ & $k$ & $q$ & $x_{i, j, k, q}$ & $i$ & $j$ & $k$ & $q$ & $x_{i, j, k, q}$ & $i$ & $j$ & $k$ & $q$ & $x_{i, j, k, q}$ & $i$ & $j$ & $k$ & $q$ & $x_{i, j, k, q}$ \\
\hline 1 & 2 & 1 & 2 & 161 & 2 & 4 & 2 & 4 & 401 & 3 & 6 & 3 & 6 & 231 & 6 & 2 & 6 & 4 & 132 \\
\hline 1 & 2 & 1 & 3 & 72 & 2 & 4 & 5 & 4 & 9 & 3 & 7 & 3 & 7 & 282 & 6 & 5 & 6 & 5 & 4 \\
\hline 1 & 2 & 1 & 4 & 89 & 2 & 4 & 6 & 4 & 132 & 4 & 2 & 4 & 1 & 125 & 6 & 5 & 7 & 5 & 3 \\
\hline 1 & 2 & 1 & 5 & 59 & 2 & 4 & 7 & 4 & 49 & 4 & 2 & 4 & 2 & 22 & 6 & 7 & 1 & 7 & 11 \\
\hline 1 & 2 & 1 & 6 & 15 & 2 & 5 & 1 & 5 & 59 & 4 & 2 & 4 & 3 & 161 & 6 & 7 & 2 & 7 & 21 \\
\hline 1 & 2 & 1 & 7 & 11 & 2 & 5 & 2 & 5 & 135 & 4 & 2 & 4 & 5 & 3 & 6 & 7 & 4 & 7 & 134 \\
\hline 1 & 2 & 1 & 8 & 38 & 2 & 5 & 3 & 5 & 4 & 4 & 6 & 4 & 6 & 360 & 6 & 7 & 5 & 7 & 26 \\
\hline 2 & 1 & 2 & 1 & 190 & 2 & 5 & 4 & 5 & 3 & 4 & 6 & 4 & 7 & 134 & 6 & 7 & 6 & 7 & 30 \\
\hline 2 & 1 & 3 & 1 & 67 & 2 & 6 & 1 & 6 & 15 & 4 & 8 & 4 & 8 & 505 & 6 & 8 & 6 & 8 & 59 \\
\hline 2 & 1 & 4 & 1 & 125 & 2 & 6 & 1 & 7 & 11 & 5 & 1 & 5 & 1 & 93 & 7 & 2 & 7 & 1 & 9 \\
\hline 2 & 1 & 5 & 1 & 13 & 2 & 6 & 2 & 6 & 36 & 5 & 2 & 5 & 1 & 13 & 7 & 2 & 7 & 2 & 153 \\
\hline 2 & 1 & 6 & 1 & 13 & 2 & 6 & 2 & 7 & 21 & 5 & 2 & 5 & 2 & 24 & 7 & 2 & 7 & 4 & 49 \\
\hline 2 & 1 & 7 & 1 & 9 & 2 & 8 & 1 & 8 & 38 & 5 & 2 & 5 & 3 & 17 & 7 & 3 & 7 & 3 & 160 \\
\hline 2 & 1 & 8 & 1 & 34 & 2 & 8 & 2 & 8 & 30 & 5 & 2 & 5 & 4 & 9 & 7 & 6 & 7 & 5 & 3 \\
\hline 2 & 3 & 1 & 3 & 72 & 2 & 8 & 3 & 8 & 50 & 5 & 6 & 5 & 6 & 40 & 7 & 6 & 7 & 6 & 50 \\
\hline 2 & 3 & 2 & 3 & 442 & 3 & 2 & 3 & 1 & 67 & 5 & 6 & 5 & 7 & 26 & 8 & 2 & 8 & 1 & 34 \\
\hline 2 & 3 & 4 & 3 & 161 & 3 & 2 & 3 & 2 & 231 & 5 & 8 & 5 & 8 & 4 & 8 & 2 & 8 & 2 & 396 \\
\hline 2 & 3 & 5 & 3 & 17 & 3 & 2 & 3 & 5 & 4 & 6 & 2 & 6 & 1 & 13 & 8 & 2 & 8 & 3 & 29 \\
\hline 2 & 3 & 6 & 3 & 131 & 3 & 2 & 3 & 8 & 50 & 6 & 2 & 6 & 2 & 315 & 8 & 4 & 8 & 4 & 185 \\
\hline 2 & 3 & 8 & 3 & 29 & 3 & 4 & 3 & 4 & 293 & 6 & 2 & 6 & 3 & 131 & 8 & 6 & 8 & 6 & 32 \\
\hline 2 & 4 & 1 & 4 & 89 & - & - & - & - & - & - & - & - & - & - & - & - & - & - & - \\
\hline
\end{tabular}

Table 9.The solution of RBP for variable $x_{i, j, k, q}$ with real flow OD in 2014

\begin{tabular}{|c|c|c|c|c|c|c|c|c|c|}
\hline $\bar{i}$ & $j$ & 1 & 2 & 3 & 4 & 5 & 6 & 7 & 8 \\
\hline & & 0 & 1 & 0 & 0 & 0 & 0 & 0 & 0 \\
\hline & & 1 & 0 & 1 & 1 & 1 & 1 & 0 & 1 \\
\hline$i$ & $j$ & 1 & 2 & 3 & 4 & 5 & 6 & 7 & 8 \\
\hline & & 0 & 1 & 0 & 1 & 0 & 1 & 1 & 0 \\
\hline & & 0 & 1 & 0 & 0 & 0 & 1 & 0 & 1 \\
\hline & & 1 & 1 & 0 & 0 & 0 & 1 & 0 & 1 \\
\hline & & 0 & 1 & 0 & 0 & 1 & 0 & 1 & 1 \\
\hline & & 0 & 1 & 1 & 0 & 0 & 1 & 0 & 0 \\
\hline & & 0 & 1 & 0 & 1 & 0 & 1 & 0 & 0 \\
\hline
\end{tabular}

Table 10. The solution of RBP for variable $y_{i, j}$ with real flow OD in 2014

The objective function value is:

$z_{2014}=\$ 5496920$ 
To verify intuitively feasibility of model, we compare the solution of RBP in 2013 with the solution of RBP in 2014 in Fig. 2. And there are only directed train in Fig. 2.

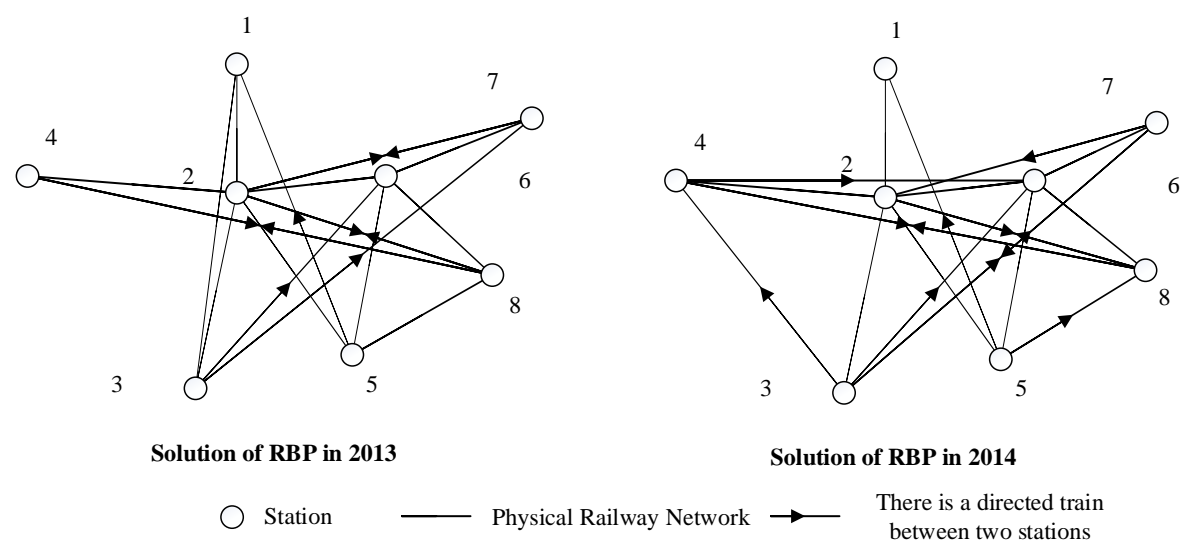

Fig. 2. The solution comparison

Comparing the solution of RBP in 2013 and 2014, the feasibility of the model can be verified from the following aspects:

1. Some directed trains are canceled.

If there are the loss of car-hours and cost, the directed train will be canceled. For example, there are 221 cars per day from station 2 to station 7 in 2013. But there are only 21 cars per day. Because of fewer flow, the directed train will cause the loss of car-hours and cost. So the directed train from station 2 to station 7 is canceled.

2. Some directed trains are built.

If the directed block can save car-hours and cost, the directed trains will be built. For example, there are 150 cars per day from station 3 to station 4 in 2013. But there are only 293 cars per day. Because of more flow, the directed train will cause the save of car-hours and cost. So the directed train from 3 to 4 is built.

The objective function of RBP model is to minimize the costs of flow traveling and delay for the train in marshalling station. So we need compare the solution with the now using RBP solution in 2014 to verify optimization of model.

The flow of transit car with resorting and transit car without resorting of station 2 in 2014 is shown in Table 11 and Table 12.

\begin{tabular}{|c|c|c|c|c|c|c|c|}
\hline Arrival leave & 1 & 3 & 4 & 5 & 6 & 7 & 8 \\
\hline 1 & 0 & 32 & 72 & 7 & 9 & 6 & 23 \\
\hline 3 & 47 & 0 & 61 & 13 & 36 & 43 & 8 \\
\hline 4 & 28 & 79 & 0 & 5 & 46 & 17 & 66 \\
\hline 5 & 5 & 1 & 2 & 0 & 1 & 0 & 0 \\
\hline 6 & 1 & 19 & 135 & 2 & 0 & 0 & 2 \\
\hline 7 & 7 & 31 & 52 & 2 & 1 & 0 & 0 \\
\hline 8 & 6 & 1 & 215 & 2 & 1 & 0 & 0 \\
\hline
\end{tabular}

Table 11. The flow of transit car with resorting in 2014 [car]

\begin{tabular}{|c|c|c|c|c|c|c|c|c|}
\hline Arrival leave & 1 & 2 & 3 & 4 & 5 & 6 & 7 & 8 \\
\hline 1 & 0 & 190 & 35 & 53 & 99 & 4 & 3 & 11 \\
\hline 2 & 161 & 0 & 231 & 22 & 24 & 315 & 153 & 396 \\
\hline 3 & 25 & 442 & 0 & 100 & 4 & 95 & 117 & 21 \\
\hline 4 & 61 & 401 & 214 & 0 & 4 & 86 & 32 & 119 \\
\hline 5 & 54 & 135 & 3 & 1 & 0 & 3 & 3 & 0 \\
\hline 6 & 14 & 36 & 212 & 225 & 38 & 0 & 50 & 30 \\
\hline 7 & 4 & 21 & 251 & 82 & 24 & 29 & 0 & 0 \\
\hline 8 & 32 & 30 & 49 & 290 & 2 & 58 & 0 & 0 \\
\hline
\end{tabular}

Table 12. The flow of transit car without resorting in 2014 [car] 
And the objective function value of real-world in the condition of the same parameters is

$z_{\text {actual }}=\$ 6527430$

Compared with the now using RBP solution in 2014, the optimization of the model can be verified from the following aspects:

1. Some directed trains are canceled.

The new solution deletes 25 directed trains, saves total 9005 car-hours per day. For example, there are 13 cars per day from station 6 to station 1 . Because of a directed block for the flow, there are 500 car-hours about car detention time under accumulation and 38 car-hours of the save time because of transit car without reclassification per day. The directed train from station 6 to station 1 causes the loss of 400 car-hours per day.

2. Volume shipped by directed trains are added.

The new solution adds volume shipped by directed trains, saves total 3538 car-hours per day.

3. Cost Saving.

The objective function value of formulation of RBP model is 5496920. And the objective function value of real-world in the condition of the same parameters is 6527430. Total cost saving is 1030510 .

4. Traffic flow adjustments.

The solution considers the balance of railway line. And some flows in busy railway line are adjusted to other rail lines to improve whole network efficiency. Such as, in existing RBP solution in 2014, the flow from station 5 to station 6 pass station 2 with reclassification operation. But in solution of RBP model, a directed block between station 5 and station 6 is built to make full use of railway between station 5 and station 6 .

\section{Conclusions}

This paper mainly focuses on Railway Blocking Problem in a network. We consider both transport cost and delay on marshaling station; and use GAMS to solve it. We give a case of 8 marshaling stations to test the model on the real world data. In the case, solution by our method can decrease $55 \%$ of car-hours and $16 \%$ of cost per day. In the meantime, we can optimize traffic flow to improve efficiency of the whole network. It is sure that our proposed models are effective, efficient and potential for application in a real world railway network.

\section{References}

[1] M Yaghini, et.al. Solving railroad blocking problem using ant colony optimization algorithm [J]. Applied Mathematical Modelling, 35(2011) 5579-5591.

[2] R.K. Ahuja, et.al. Solving Real-Life Railroad Blocking Problems [J]. Interfaces, 37(2007) 404-419.

[3] M Yaghinia and R Akhavan. Multicommodity Network Design Problem in Rail Freight

Transportation Planning [J]. Procedia - Social and Behavioral Sciences, 43(2012) 728-739.

[4] C Barnhart, et.al. Railroad Blocking: A Network Design Application [J]. Operations Research, 48(2000) 603-614.

[5] Ahuja, et.al. Network Models in Railroad planning and scheduling [J]. Operation Research, 1(2005) 54-101.

[6] A Balakrishnan, et.al. A Dual-ascent Procedure for Large-scale Uncapacitated Network Design [J]. Operations Research, 73(1989) 716-740.

[7] H.Xu, et al. Study on the Model and Algorithm of the Formation Plan of Single Group Trains at Technical Service Stations (In Chinese) [J]. Journal of the China Railway Society, 28(2006) 12-17. 
[8] S.Yang, et al. An Artificial Neural Network Method for Marshalling Plan (In Chinese) [J]. Journal of Changsha Railway University, 20(2002) 79-84.

[9] X.Li. Study on Optimization of Marshalling Plan and Flow Path Based on Uncertain Parameters (In Chinese) [D]. Southwest Jiaotong University, 2002.

[10] H.N. Newton. Network Design under Budget Constraints with Application to the Railroad Blocking Problem [D]. Auburn University, 1996.

[11] H.N. Newton, et.al. Constructing Railroad Blocking Plans to Minimize Handling Costs [J]. Transportation Science, 32(1998) 330-345.

[12] L.Bodin, et.al. A Model for the Blocking of Trains [J]. Transportation Research Part B Methodological, 14(1980) 115-120.

[13] M.Yaghini, et.al. Solving Railroad Blocking Problem Using Ant Colony Optimization Algorithm [J]. Applied Mathematical Modelling, 35(2011) 5579-5591.

[14] Y.Yue, et.al. Multi-route Railroad Blocking Problem by Improved Model and Ant Colony Algorithm Real World [J]. Computers \& Industrial Engineering, 60(2011) 34-42.

[15] A.Brooke, et.al. GAMS Language Guide. 2006. 